\title{
Fortalecimiento del capital económico del ejido forestal Agostadero de Aguirre, Chihuahua
}

Strengthening the economic capital of the forest ejido Agostadero de Aguirre, Chihuahua

Rascón-Solano Joel $^{1}$, Kiessling-Davison Christian Mauricio ${ }^{1}$, Villarreal-Ramírez Víctor Hugo ${ }^{1 凶}$, Ma. Guadalupe Macias Lopez ${ }^{1}$, Jose Javier Hermosillo Nieto ${ }^{1}$.

${ }^{1}$ Facultad de Ciencias Agrícolas y Forestales. Universidad Autónoma de Chihuahua. Km 2.5 carretera Delicias-Rosales, C. P. 33000, Delicias, Chihuahua, México

${ }^{凶}$ Autor para correspondencia: vvillar@uach.mx

Recibido: 28/09/2019

Aceptado: 25/11/2019

\section{RESUMEN}

El ejido Agostadero de Aguirre cuenta con 21,936.68 metros cúbicos rollo total árbol ( $\left.\mathrm{m}^{3} \mathrm{RTA}\right)$ del género Pinus distribuidos en 15 anualidades de explotación, sin embargo, se encuentra presente en el mercado de venta de madera rolliza ya que no posee equipo de asierre. El objetivo en el presente estudio es analizar el potencial económico alcanzado por el ejido mediante la adquisición de equipo de transformación y comercialización regional de productos forestales maderables. Se concidera la adquisición de equipos de asierre de bajo rendimiento por un costo total de instalación y puesta en marcha por $\$ 1,123,495.00 \mathrm{MN}$ bajo un financiamniento del \% 70 a una tasa de interes del \% 12 anual. En términos financieros se concluye que el proyecto es rentable debido a que cuenta con un VAN de 1,852,120.19, una TIR superior en 49.11 puntos porcentuales a la TREMA establecida del $10 \%$ y una Rel. B/C de 1.29. En cuanto a los indicadores economicos evaluados se determinó un P.E. de 45,943.89 PT producidos y un P.R. de 1.67 años en base a un horizonte de cinco años, sin embargo, es necesario evaluar el mercado potencial mediante la compra de materia prima externa al ejido.

Palabras clave: Pinus, Equipo de asierre, Financieros, Rentable.

\begin{abstract}
The ejido Agostadero de Aguirre has 21,936.68 cubic meters total roll tree of the genus Pinus distributed in 15 operating annuities, however, It is present in the market for the sale of roundwood since it does not have sawmill. The objective in the present study is to analyze the economic potential achieved by the ejido through the acquisition of processing equipment and regional marketing of timber forest products. The acquisition of a low-performance sawmill is considered for a total installation and commissioning cost of $\$ 1,123,495.00 \mathrm{MN}$ under a financing of $\% 70$ at an annual interest rate of $\% 12$. In financial terms, it is concluded that the project is profitable because it has a NPV of 1,852,120.19, an IRR of 49.11 percentage points higher than the established Annual Average Rate of Return of $10 \%$ and a Relief Benefit/Cost of 1.29. As for the economic indicators evaluated, and P.E. of 45,943.89 PT
\end{abstract}


produced and a P.R. 1.67 years based on a five-year horizon, however, it is necessary to evaluate the potential market by purchasing raw material external to the ejido.

Keywords: Pinus, Sawmill, Financial, Profitable.

\section{INTRODUCCIÓN}

El ejido Agostadero de Aguirre cuenta con una superficie total de 14,549.75 hectáreas (ha), el área total bajo manejo forestal es 4,761.25 ha, con extensión aprovechable en producción de 2,306.00 ha. El volumen total de remoción de las áreas de extracción es de 21,936.68 metros cúbicos rollo total árbol $\left(\mathrm{m}^{3} \mathrm{RTA}\right)$ del género Pinus distribuidos en 15 anualidades de explotación. El principal mercado de madera en rollo se encuentra en a la ciudad de Hidalgo del Parral, ya que el ejido no cuenta con equipo de asierre o trasformación (Rascón-Pérez \& Rascón-Solano, 2018).

Según lo descrito por Bedoya-Hernández \& Loaiza-Parra (2011) al formular una propuesta clara para el fortalecimiento de una empresa, se debe analizar primero si ésta generará utilidades a corto, mediano y largo plazo, para de esta forma generar bienes o servicios útiles para el ser humano y la sociedad en general (MartínezFierro et al., 2014), los resultados permiten

\section{MATERIALES Y MÉTODOS}

El estudio se realizó en el ejido Agostadero de Aguirre del municipio de Balleza, Chihuahua, el establecimiento del centro industrial para la transformación de productos forestales maderables se contempló en un terrritorio de uso común a 350 metros del tramo carretero Guachochi - Hidalgo del Parral, a 65 kilometros de distancia de la primera población, con coordenadas al centro del polígono 2655'58.84" Latitud Norte 10643'39.88" Longitud Oeste. apreciar que el proyecto generará beneficios importantes y se mantendra competente en el mercado (Rascón-Solano, 2018).

Previo a la evaluacion es necesario analizar el conjunto de conceptos mediante los cuales se identifican los costos (CITEmadera, 2009), elaborar proyecciones y corridas financieras genera la información elemental para determinar la factibilidad de un proyecto planteado para incrementar las condiciones economicas de una empresa (Mejía-Saavedra et al., 2013), Luján-Álvarez et al. (1990) recomiendan llevar a cabo un control en el registro de información contable administrativo en cada fase del proceso de formulación y evaluación de los proyectos de inversión forestal.

El objetivo en el presente estudio es analizar el potencial económico alcanzado por el ejido Agostadero de Aguirre mediante la adquisición de equipo transformación y comercialización regional de productos forestales maderables del género Pinus.

El funcionamiento del centro de asierre se estimó mediante el análisis de cadenas productivas instaladas en la región efectuado por Rascó-Solano (2018) con rendimientos medios de producción en un $50 \%$ de coeficiente de asierre. El ejido cuenta con posibilidades de aprovechamiento anuales por 1,462 $\mathrm{m}^{3}$ RTA, con un $85 \%$ de produto aserrable, de los cuales $869.89 \mathrm{~m}^{3}$ corresponden a largas dimesiones equivalentes a un estimado de 138,224.04 Pies Tablares (PT), $217.47 \mathrm{~m}^{3}$ correspondientes a asierre de cortas dimensiones, un periodo de actividad de 45.78 
días por año, con producción de 4,025.24 PT diarios.

Rascón-Solano (2019) menciona en su investigación que para el sector forestal comunmente se emplea para la evaluación de proyectos la metodología desarrollada por Baca-Urbina (2013), el método emplea indicadores financieros como el cálculo del Valor Actual Neto (VAN), la Tasa Interna de Retorno (TIR) y la Relación Beneficio/Costo (Rel. B/C); para mejorar la calidad de los resultados es necesario determinar el Periodo de Recuperación de la inversión (PR) y el Punto de Equilibrio (PE).

Respecto al análisis financiero para el ejido se empleó la metodología de formulación y evaluación de proyectos de inversión. Mediante el cálculo del Valor Actual Neto es posible considerar el flujo de efectivo a través del tiempo.

$$
V A N=-I_{0}+\sum_{t=1}^{n} \frac{F t}{(1+k) t}=-I_{0}+\frac{F 1}{(1+k)}+\frac{F 2}{(1+k)^{2}}+\cdots+\frac{F n}{(1+k)^{n}}
$$

La Tasa Interna de Retorno es la tasa de interés o rentabilidad que ofrece una inversión, indica el porcentaje de descuento que se aplica a los valores de los flujos de los beneficios e inversión para el pago de inversiones (Calderón-García et al., 2018), para evaluar este indicador se empleará una Tasa de Rendimiento Media Anual (TREMA) del $10 \%$.

$$
T I R=\sum_{T=0}^{n} \frac{F n}{(1+i)^{n}}=0
$$

La Relación Beneficio/Costo es el cálculo que permite analizar el efecto que tienen los ingresos de la actividad sobre los egresos de la misma, el resultado de este ejercicio será aceptado siempre y cuando el resultado sea mayor a uno.

$$
\text { Rel. }{ }^{B} /_{C}=\frac{\text { Ingresos actualizados }}{\text { Egresos actualizados }}
$$

Para evaluar la factibilidad económica del proyecto se empleó el criterio para el cálculo del punto de equilibrio, este indica la cantidad de productos que es necesario generar para que "el ingreso total de la empresa sea igual a sus gastos totales" (Mazón-Arevalo et al. 2017). 


$$
\text { P.E. }=\frac{C F}{P-C V}
$$

Según Canales-Salinas (2015) el periodo de recuperación frecuentemente se emplea en las evaluaciones económicas de los proyectos de inversión, este periodo será aceptado solo si el tiempo trascurrido en recuperar el efectivo en inversión es inferior al horizonte establecido para el proyecto.

$$
P R=\sum_{n=0}^{T p} \frac{F_{j}}{(1+i)^{n}}
$$

\section{RESULTADOS Y DISCUSIÓN}

De acuerdo con los conceptos analizados para los costos de la madera en rollo de pino en el Cuadro 1 se determina que, el ejido tendra que generar un gasto estimado en \$ 5,200.00 Moneda Nacional (MN) por millar de pies tablares extraidos de las áreas de corta, en general el costo total expedido por la administración ejidal sera de \$958,353.39 MN aproximadamente para las anualidades de explotación.

Cuadro 1. Costos determinados para la extracción y abastecimiento de madera rolliza de Pinus.

\begin{tabular}{lrr}
\hline \multicolumn{1}{c}{ Concepto } & Costo/Pie Doyle (\$) & Costo/Millar PD (\$) \\
\hline Madera en pie & 1.20 & $1,200.00$ \\
Derribo y troceo & 0.25 & 250.00 \\
Limpia forestal & 0.15 & 150.00 \\
Arrastre, junta y carga & 0.85 & 850.00 \\
Documentador y montero & 0.05 & 50.00 \\
Planeación de caminos & 0.20 & 200.00 \\
Flete de trocería & 2.50 & $2,500.00$ \\
\hline \multicolumn{1}{c}{ Costos Totales } & 5.20 & $5,200.00$
\end{tabular}

De acuerdo con lo establecido en el DOF (2018) el monto del salario mínimo para el área de Salarios Mínimos Generales será de \$ 102.68 pesos mexicanos diarios por jornada laboral, atendiendo la reglamentación se determinó como ingreso mínimo diario \$ 30.00
MN para los auxiliares del centro de asierre, de acuerdo con la producción diaria estimada, los auxiliares de operación obtendrán un ingreso de \$ 120.75 MN por jornada laboral.

En el Cuadro 2 se muestran los ingresos obtenidos por los operadores, auxiliares y personal administrativo del centro de asierre, se calculó un gasto anual por $\$ 103,207.29 \mathrm{MN}$ 
como concepto de nómina. De acuerdo con las condiciones del equipo de asierre propuesto para el ejido, será necesario integrar multitareas en algunos de los puestos de trabajo debido a la baja producción maderable.

Cuadro 2. Salarios establecidos para el centro de asierre.

\begin{tabular}{lrr}
\hline \multicolumn{1}{c}{ Concepto } & Costo/Pie Tablar $\mathbf{( \$ )}$ & Costo/Millar PT $\mathbf{( \$ )}$ \\
\hline Oficinista y jefe de asierre & 0.05 & 50.00 \\
Documentador & 0.03 & 30.00 \\
Rodador & 0.03 & 30.00 \\
Aserrador & 0.06 & 60.00 \\
Volteador & 0.03 & 30.00 \\
Desorillador & 0.04 & 40.00 \\
Auxiliar desorillador & 0.03 & 30.00 \\
Cabeceador & 0.04 & 40.00 \\
Auxiliar cabeceador & 0.03 & 30.00 \\
Clasificador y baño químico & 0.04 & 40.00 \\
Dos apiladores & 0.06 & 60.00 \\
Palillero y recortador & 0.06 & 60.00 \\
Tabletero y recortador & 0.06 & 60.00 \\
\hline \multicolumn{1}{c}{ Costos Totales } & 0.56 & 560.00
\end{tabular}

Generalmente las industrias forestales de la region sur de la Sierra Madre Occidental de Chihuahua emplean conceptos poco convencionales para estipular los costos administrativos ejidales y para los centros de asierre, estos conceptos se muestran en el Cuadro 3 estableciendo los montos a pagar por millar de pies tablares producidos y comercializados.

Servicios establecidos como el concepto de pago por servicios técnicos da referencia a la cuota establecida a cubrir por el manejo sustentable de los bosques bajo explotación, el pago de servicios a (Unidad de Manejo Forestal Regional 0807) UMAFOR corresponde al monitoreo y combate de incendios forestales dentro del ejido, por ultimo, se efectua el pago de servicios a la Unidad de Silvicultores Unidos de Guachochi por concepto de producción de planta empleada en las reforestaciones del ejido.

A diferencia de industrias de la región se integran los conceptos de pago se seguro a ejidatarios y el pago por un seguro industrial contra catastros para la industria a establecer en el ejido.

Cuadro 3. Costos calculados por el pago de servicios y administración. 
Rascón-Solano et al., 2019

\begin{tabular}{lcr}
\hline \multicolumn{1}{c}{ Concepto } & Costo/Pie Tablar $\mathbf{( \$ )}$ & Costo/Millar PT $\mathbf{( \$ )}$ \\
\hline Servicios técnicos & 0.20 & 200.00 \\
Servicios UMAFOR & 0.04 & 40.00 \\
Servicios silvicultores & 0.04 & 40.00 \\
Presidente comisariado & 0.08 & 80.00 \\
Suplente presidente & 0.03 & 30.00 \\
Secretario & 0.04 & 40.00 \\
Suplente secretario & 0.03 & 30.00 \\
Tesorero & 0.04 & 40.00 \\
Suplente tesorero & 0.03 & 30.00 \\
Consejo de vigilancia & 0.04 & 40.00 \\
Suplente consejo & 0.03 & 30.00 \\
Velador & 0.04 & 40.00 \\
Seguro a ejidatarios & 0.09 & 92.00 \\
Seguro Industrial & 0.21 & 210.00 \\
Luz Agua & 0.15 & 150.00 \\
Costos Totales & 0.03 & 30.00 \\
\hline \multicolumn{1}{c}{$\quad 1.12$} & $1,122.00$
\end{tabular}

De acuerdo con lo descrito por KiesslingDavison \& Licón-Trillo (2005) en el estado de Chihuahua se maneja como clasificación para la madera aserrada, 2 y mejor para maderas moldurables, número 3 para madera empleada en elaboracion de puertas y ventanas, número 4 y número 5 empleada como madera de construcción. Generalemente las dimenciones en gruesos producidas en el estado son 7/8,5/4 y 6/4 para madera aserrada de largas dimensiones (Luján-Álvarez, 1990).

En el Cuadro 4 se muestran los costos de los productos generados, las necesidades actuales del mercado de la madera aserrada han incrementado el consumo de productos como es el Polín empleado en la costrucción y el Pallet ampliamente utilizado por las industrías manufactureras.

Cuadro 4. Valor de los produtos, costos de transformación y su distribucion de producción.

\begin{tabular}{|c|c|c|c|}
\hline Clase de madera & Porcentaje (\%) & Precio (\$) & Costo producción (\$) \\
\hline 2 y Mejor $7 / 8$ & 2.63 & 17.68 & 6.88 \\
\hline Número $37 / 8$ & 13.13 & 14.02 & 6.88 \\
\hline Número $47 / 8$ & 18.38 & 12.48 & 6.88 \\
\hline Número $57 / 8$ & 18.38 & 9.29 & 6.88 \\
\hline Número $45 / 4$ y $6 / 4$ & 7.50 & 12.48 & 6.88 \\
\hline Número 5 5/4 y 6/4 & 7.50 & 9.29 & 6.88 \\
\hline Polín & 7.50 & 9.29 & 6.88 \\
\hline Pallet & 25 & 8.05 & 6.88 \\
\hline Tota & 100 & 92.58 & 55.06 \\
\hline
\end{tabular}


De acuerdo con la posibilidad anual de madera del género Pinus del ejido Cieneguita de la Barranca se contempla la adquisición de equipos de asierre de producción media, el equipo adecuado para la producción estimada es principlamente un aserradero tipo sierra- banda con cinta de sesis pulgadas, de acuerdo con las indicaciones del fabricante, este equipo tiene la capacidad de aserrar un maximo de 8,000 PT por jornada laboral, en la Figura 1 se muestra la sierra-banda con valor de venta \$ 371,200.00 MN.

Figura 1. Aserradero portátil, fabricado por Taller de Soldadura y Maquinaria Forestal NEKS.

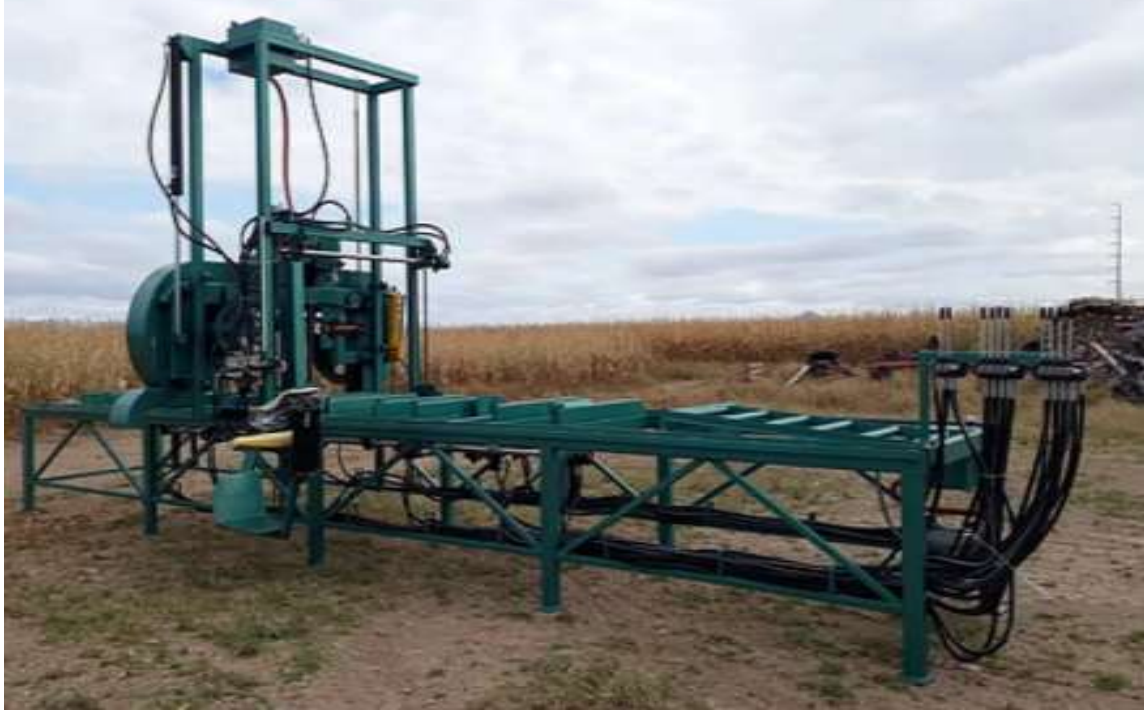

Fuente: https://www.maquinariaforestal.com.mx/aserradero-portatil

En el Cuadro 5 se especifican los equipos que la empresa ejidal ha conciderado adquirir para lograr la transforación de sus productos maderables, los costos establecidos se conceptualizan de acuerdo a los precios del fabricante tomando en cuenta los módulos de transformación y su instalación en el ejido, la administración ejidal contempla cubrir el $30 \%$ del monto total, solicitando el resto de la inversión por medio de financiamiento a una tasa de interes del \% 12. Adicionalmente se concidera el costo fijo de inversión por \$ 79,826.94 MN por concepto del costo de la tierra. 
Cuadro 5. Costo del estableciemiento de la industria forestal para madera aserrada.

\begin{tabular}{|c|c|c|}
\hline Concepto & Cantidad & Costo (\$) \\
\hline Infraestructura y electricidad & 1 & $400,000.00$ \\
\hline Aserradero portátil & 1 & $371,200.00$ \\
\hline Desorilladora & 1 & $64,960.00$ \\
\hline Cabeceadora & 1 & $33,640.00$ \\
\hline Mesa de roles de 8 pies & 5 & $19,000.00$ \\
\hline Equipo de protección & 15 & $12,460.00$ \\
\hline Equipo contra incendios & 5 & $5,165.00$ \\
\hline Oficina y equipo de computo & 1 & $52,350.00$ \\
\hline Tabletera & 1 & $63,800.00$ \\
\hline Palillera & 1 & $44,080.00$ \\
\hline Recortador & 2 & $56,840.00$ \\
\hline
\end{tabular}

El primer indicador generado para evaluar la rentabilidad del proyecto de inversión es el Valor Actual Neto, el cual de acuerdo con la ecuación descrita anteriormente concibe un resultado de 1,852,120.19, este flujo de efectivo al ser positivo se considera aceptable para la estabilidad de la empresa.

La Tasa Interna de Retorno es aceptable si cuenta con un valor positivo resultante mayor a 0 , de acuerdo con la TREMA establecida anteriormente, se determina que el proyecto de inversión es un $49.11 \%$ mayor a la tasa de rendimiento mínima establecida y por lo tanto se acepta como viable.

La Relación Beneficio/Costo del proyecto arroja como resultado un valor de 1.29 , esto indica que el valor es mayor a uno y por lo tanto es aceptado, en cuanto a las inversiones y utilidades de la empresa quiere decir que por cada unidad invertida en el proyecto se obtendrán \$ 0.29 MN de ganancia.

De acuerdo con los indicadores determinados por Baca-Urbina (2013) el proyecto resulta ser viable y puede ejecutarse, ya que los indicadores resultan ser positivos en la totalidad de los casos evaluados.

De acuerdo con los datos resultantes en la estimación del Punto de Equilibrio, la producción total se ubica en 45,943.89 pies tabla producidos por los diversos productos aserrados equivalentes al \% 24.93 de la posibilidad de transformación total anual, a continuación, se muestra en la Figura 2 el comportamiento de los costos fijos, variables y totales frente a la utilidad esperada. (BacaUrbina, 2013) 
Figura 2 . Grafica del cálculo del Punto de Equilibrio de la producción maderable.

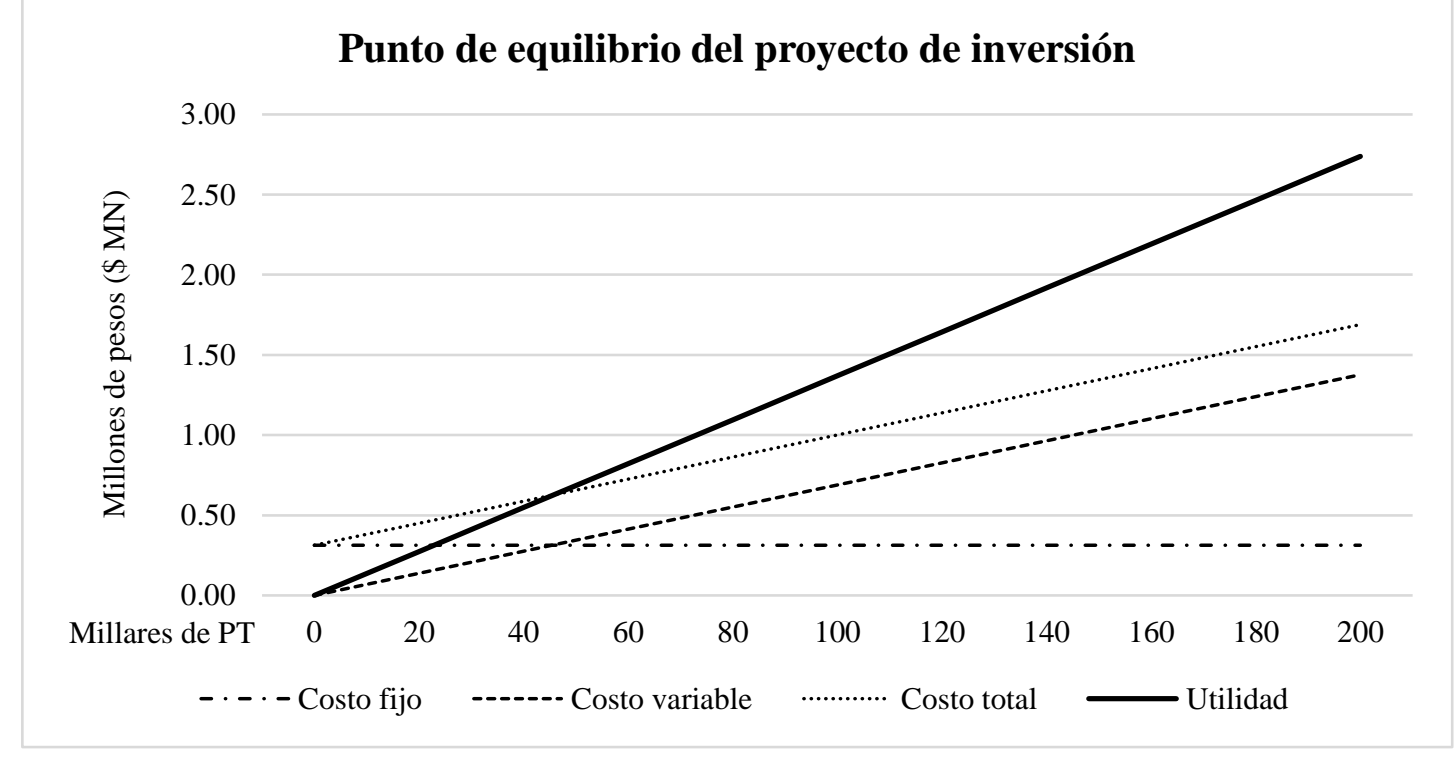

Con los cálculos realizados se estimo un Periodo de Recuperación de la inversión de 1.67 años equivalentes a mantener un periodo de transformación y comercialización de productos aserrados de un año y ocho meses, este indicador genera la confiabilidad para que el proyecto sea ejecutado.

De acuerdo con los indicadores económicos analizados en este proyecto de inversión se plantea viable la adquisición de equipos de asierre e infraestructura necesarios, el ejido Agostadero de Aguirre a pesar de contar con una posibilidad de aprovachamiento baja obtendrá un ingreso superior al actual.

\section{CONCLUSIONES}

Los indicadores financieros y económicos evaluados muestran que la tecnificación del ejido es viable y rentable, en su totalidad los datos resultantes son favorables para la puesta en marcha del proyecto de inversión y se consideran estables mientras se sigan los lineamientos estipulados en la investigación.
La rentabilidad del proyecto de inversión forestal para el ejido Agostadero de Aguirre incrementaría considerablemente el reparto de utilidades entre los ejidatarios, mejorando las condiciones de vida de los mismos y las condiciones administrativas de la empresa.

La adquisición de equipos de asierre puede mejorar las condiciones economicas de la empresa, de esta forma en el mediano plazo el ejido puede concretar la compra de madera en rollo a comunidades vecinales, ya que algunas no cuentan con equipos de transformación.

Es necesario que la empresa efectue un estudio de mercado de productos aserrados, de esta forma puede generar la información adecuada para consolidar mejores ventas de madera aserrada, sin embargo la baja producción con la que contará en el corto plazo no le permitiría posicionar sus productos con consumidores potenciales. 


\section{LITERATURA CITADA}

Baca-Urbina, G. (2013). Evaluación de proyectos (Séptima edición ed.). Cd. de México, CDMX, México: Editorial Mc Graw Hill.

Bedoya-Hernández, P. A., \& Loaiza-Parra, Y. P. (2011). Estudio de factibilidad para la creación de la empresa "Empleos especializados LTDA" en la ciudad de Perira. Tesis de Licenciatura. Cd. de Pereira, Pereira, Colombia: Universidad Tecnológica de Pereira, Facultad de ingeniería industrial.

Calderón-García, D. M., Olivas-García, J. M., Legarreta-González, M. A., CarrilloSotelo, M. E., Anchondo-Nájera, J. Á., \& Luján-Álvarez, C. (Julio/Septiembre de 2018). Funcionamiento y viabilidad económica de un módulo de producción acuapónico de los Mochis, Sinaloa, México. Custos e @gronegócio on line, Vol. 14(No. 3), 131-146.

Canales-Salinas, R. J. (Enero-Junio de 2015). Criterios para la toma de decisión de Inversiones. Revista Electrónica de Investigación en Ciencias Económicas, Vol. 3(No. 5), 101-117. https://doi.org/10.5377/reice.v3i5.2022

CITEmadera. (2009). Costos en la producción de muebles y carpintería en madera. En C. G. Jessica Moscoso, Costos en la producción de muebles y carpintería en madera. (pág. 50 páginas). Lima, Perú: Viceministerio de MYPE e IndustriaMinisterio de la Producción.

DOF. (2018). RESOLUCIÓN del H. Consejo de Representantes de la Comisión Nacional de los Salarios Mínimos que fija los salarios mínimos general y profesionales vigentes a partir del 1 de enero de 2019. Cámara de Diputados del H. Congreso de la Unión, Secretaría Genera, Secretaría de Servicios Parlamentarios, Diario Oficial de la Federación (DOF), Cd. de México, CDMX, México.

Kiessling-Davison, C. M., \& Licón-Trillo, L. P. (2005). Apuntes de industrias forestales. Cd. Delicias, Chihuahua, México: Universidad Autónoma de Chihuahua, Facultad de Ciencias Agrícolas y Forestales.

Luján-Álvarez, C. (1990). Determinación del patrón óptimo de producción del aserradero "Las Delicias" del ejido forestal "La Trinidad" municipio de Guadalupe y Calvo, Chihuahua. Tesis de Maestría. Cd. Delicias, Chihuahua, México: Universidad Autónoma de Chihuahua, Facultad de Ciencias Agrícolas y Forestales.

Martínez-Fierro, C. P. (2014). Estudio de factibilidad sobre la engorda de ganado bovino de carne para la asociación ganadera de Camargo, Chihuahua. Estudio de Caso de Maestría. Cd. Delicias, Chihuahua, México: Universidad Autónoma de Chihuahua, Facultad de Ciencias Agrícolas y Forestales.

Mazón-Arevalo, L., Villao-Burgos, D., Núñez, W., \& Serrano-Luyó, M. (Junio de 2017). Análisis de punto de equilibrio en la toma de decisiones de un negocio: caso Grand Bazar Riobamba - Ecuador. Revista de Estrategias del Desarrollo Empresarial, Vol. 3(No. 8), 14-24.

Mejía-Saavedra, M. I. (2013). Proyecto de inversión: Modernización de la granja 
lechera de la Facultad de Ciencias Agrícolas y Forestales en Cd. Delicias, Chihuahua. Estudio de Caso de Maestría. Cd. Delicias, Chihuahua, México: Universidad Autónoma de Chihuahua, Facultad de Ciencias Agrícolas y Forestales.

Rascón-Pérez, J., \& Rascón-Solano, J. (2018). Programa de manejo forestal maderable del ejido Agostadero de Aguirre, Balleza, Chihuahua. Cd. Guachochi, Chihuahua, México: Secretaría del Medio Ambiente y Recursos Naturales.

Rascón-Solano, J. (2018). Mejoramiento de la rentabilidad de la industria forestal en la empresa ejidal Aboreachi, Guachochi, Chihuahua. Estudio de Caso de Maestría Cd. Delicias, Chihuahua, México: Universidad Autónoma de Chihuahua, Facultad de Ciencias Agrícolas y Forestales.

Rascón-Solano, J. (2019). Proyecto de inversión para una empresa forestal del municipio de Guachochi, Chihuahua. Tesis de Maestría. Cd. Delicias, Chihuahua, México: Universidad Autónoma de Chihuahua, Facultad de Ciencias Agrícolas y Forestales.

Copyright (c) 2019 Joel Rascón-Solano, Christian Mauricio Kiessling-Davison, Victor Hugo Villarreal-Ramirez, Maria Guadalupe Macias López y José Javier Hermosillo Nieto

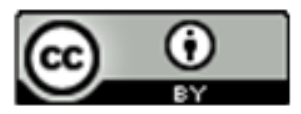

Este texto está protegido por una licencia licencia CreativeCommons 4.0.

Usted es libre para Compartir — copiar y redistribuir el material en cualquier medio o formato- y Adaptar el documento —remezclar, transformar y crear a partir del material- para cualquier propósito, incluso para fines com erciales, siempre que cumpla la condición de:

Atribución: Usted debe dar crédito a la obra original de manera adecuada, proporcionar un enlace a la licencia, e indicar si se han realizado cambios. Puede hacerlo en cualquier forma razonable, pero no de forma tal que sugiera que tiene el apoyo del licenciante o lo recibe por el uso que hace de la obra.

Resumendelicencia - Textocompletodelalicencia 